\title{
Regular Cayley maps for finite abelian groups
}

\author{
Marston Conder · Robert Jajcay • Thomas Tucker
}

Received: 8 May 2006 / Accepted: 5 September 2006 /

Published online: 4 October 2006

(C) Springer Science + Business Media, LLC 2006

\begin{abstract}
A regular Cayley map for a finite group $A$ is an orientable map whose orientation-preserving automorphism group $G$ acts regularly on the directed edge set and has a subgroup isomorphic to $A$ that acts regularly on the vertex set. This paper considers the problem of determining which abelian groups have regular Cayley maps. The analysis is purely algebraic, involving the structure of the canonical form for $A$. The case when $A$ is normal in $G$ involves the relationship between the rank of $A$ and the exponent of the automorphism group of $A$, and the general case uses Ito's theorem to analyze the factorization $G=A Y$, where $Y$ is the (cyclic) stabilizer of a vertex.
\end{abstract}

Keywords Regular map · Cayley graph · Abelian group

\section{Introduction}

For the purposes of this paper, a map $M$ is an embedding of a connected graph in an orientable closed surface such that each component (or "face") of the complement of the graph is homeomorphic to an open disc. An automorphism of $M$ is an isomorphism of the vertex-edge-face structure of $M$ induced by an orientation-preserving

Supported in part by the N.Z. Marsden Fund (grant no. UOA0124).

M. Conder $(\bowtie)$

Department of Mathematics, University of Auckland, Private Bag 92019, Auckland, New Zealand

e-mail: m.conder@auckland.ac.nz

\section{R. Jajcay}

Department of Mathematics and Computer Science, Indiana State University, Terre Haute,

IN 47809 USA

e-mail: jajcay@cayley.indstate.edu

T. Tucker

Mathematics Department, Colgate University, Hamilton, NY 13346, USA

e-mail: ttucker@mail.colgate.edu 
homeomorphism of the surface. Equivalently, a map automorphism is an automorphism of the underlying graph which preserves the cyclic order of edges incident to each vertex given by a fixed orientation of the surface. We denote by $\operatorname{Aut}(M)$ the group of all automorphisms of $M$. (Note that in other contexts, one may consider orientationreversing homeomorphisms, or embeddings in non-orientable surfaces, but we do not do that here.)

A map $M$ is called regular (or sometimes rotary, or orientably-regular) if $\operatorname{Aut}(M)$ acts transitively on the set of directed edges of the map. Since any automorphism that fixes a directed edge fixes also the incident vertices and faces, and hence by connectivity, leaves the whole map fixed, any such transitive action is sharply transitive (or regular), with trivial stabilizers. For an orientable map, being regular is equivalent to having for every vertex $v$ an automorphism that rotates the edges incident to $v$ in single steps around $v$, and for every edge $e$ an automorphism that reverses the direction of $e$ (essentially rotating it 180 degrees about its midpoint). For example, the Platonic solids are regular maps. More on regular maps is found in [6, 7, 18, 24].

A Cayley map [1] for a group $A$ is a map $M$ such that $\operatorname{Aut}(M)$ has a subgroup isomorphic to $A$ acting regularly on the vertex set. By [19], this means the underlying graph of the map, under the action of $A$, is a Cayley graph for the group $A$; a Cayley graph for $A$ with generating set $X$ has $A$ as vertex set with a directed edge from $a$ to $a x$ for every $a$ in $A$ and $x$ in $X$. (In the undirected graph underlying a Cayley graph, we identify pairs of parallel edges caused by involutions in $X$.) Moreover, the action of $A$ on the vertex set is left multiplication, so the cyclic ordering of generators $X$ and their inverses must be the same at every vertex of the map. Thus, a Cayley map for the group $A$ is a strongly symmetric embedding of a Cayley graph for $A$, in the language of [23], or the derived graph of a one-vertex voltage graph with a directed edge for each generator in $X$, in the language of [9]. For a detailed development of the theory of Cayley maps, including their recognition from a combinatorial description of a map, their relationship to planar tessellations, and their role as universal regular coverings, we cite [18], which forms much of the motivation for this paper.

Generally, we are interested in determining which finite groups $A$ have a regular Cayley map, but in this paper we consider only abelian groups. The problem is purely algebraic, and divides naturally into "balanced" and "unbalanced" cases, depending on whether or not $A$ is normal in $G=\operatorname{Aut}(M)$. The balanced case requires the existence of an automorphism $f$ of the group $A$ and an element $x$ of $A$ whose orbit under $f$ generates $A$ and contains the inverse of $x$. We show how the question reduces to finding such automorphisms for the Sylow $p$-subgroups of $A$ having compatible orders; the case $p=2$ is particularly difficult. We obtain a number of sufficient conditions for $A$ to have a balanced regular Cayley map: for example, all abelian groups of odd order do. We also obtain necessary conditions involving the rank of $A$ and the exponent of its automorphism group, which allow us to construct large classes of abelian groups that fail to have a balanced regular Cayley map. The unbalanced case involves a detailed analysis of the factorization of the automorphism group $G$ as $A Y$ where $Y$ is cyclic, involving both Ito's theorem [11] and its recent generalization [5]. We obtain a necessary condition that $A$ be a cyclic or $\mathbb{Z}_{2}$-by-cyclic extension of a balanced group, and this condition allows us to construct infinite classes of abelian groups having no regular Cayley map, balanced or unbalanced. 
We note that even small groups can provide significant obstacles. For example, we show that the only cyclic groups failing to have a nontrivial regular Cayley map are those of order $2^{k} n$ where $n$ is a product of distinct Fermat primes, one of which is 3 if $k=0$ or 1 . When we began this research, we could not show this even for $\mathbb{Z}_{15}$ without an exhaustive computer search. As another example, to show that $\mathbb{Z}_{2} \times \mathbb{Z}_{4} \times \mathbb{Z}_{8}$ has no regular Cayley map requires most of the results of this paper.

Since the original announcement of our results and the appearance of a preprint of this paper, Mikhail Muzychuk [16, 17] has provided necessary and sufficient conditions for an abelian group $A$ to have a regular Cayley map, in both the balanced and unbalanced cases. Even in the balanced case, the conditions are highly technical; for example, it is not clear how to use them to determine efficiently (such as in polynomial time) whether a given finite abelian 2-group $A$ has a balanced regular Cayley map.

The organization of this paper is as follows. In Section 2, we place the problem in a purely algebraic setting and relate that setting to the viewpoint of skew morphisms and balanced Type I/Type II Cayley maps developed by Širáň and Škoviera [21]. We also show that many nonabelian groups, for example all finite nonabelian simple groups, have balanced regular Cayley maps, and that some abelian groups have only unbalanced regular Cayley maps. In Sections 3 and 4, we consider balanced regular Cayley maps, first for general groups and abelian groups of odd order, and then for abelian 2-groups. In Section 5, we use Ito's theorem to give a structural description of the automorphism group of a regular Cayley map, and in Section 6, we use this to construct infinitely many abelian 2-groups having no regular Cayley map. Finally in Section 7, we ask some questions that arise naturally from earlier sections and are not answered directly by Muzychuk's work.

We would like to thank Cai Heng Li who pointed out to us the usefulness of Ito's theorem, Dan Saracino and Al Hales for helpful discussions about the orders of automorphisms of abelian groups, and Laci Kovacs and Mike Newman for their advice about duality on the subgroup lattice of a finite abelian group.

\section{The algebra of regular Cayley maps}

By connectivity, the automorphism group of a regular map is generated by a rotation $\rho$ at any vertex $v$ and an edge reversal $\lambda$ along any edge $e$ incident to $v$. Conversely, given any group $G=\langle\rho, \lambda\rangle$, where $\lambda$ is an involution, we can construct a regular map $M$ with $\operatorname{Aut}(M)=G$ : the vertices are the left cosets of $\langle\rho\rangle$, the undirected edges are the left cosets of $\langle\lambda\rangle$, the faces are the left cosets of $\langle\rho \lambda\rangle$, and incidence is given by non-empty intersection of cosets. Directed edges can be labelled by elements of $G$.

Hence, a question about regular maps is really a question about groups generated by two elements, one of which is an involution. This observation is an old one, and has been exploited in the construction of families of regular maps and the enumeration of small regular maps (see $[4,15,20]$ for example). We make the same observation for regular Cayley maps for a group $A$. A group $G$ factorizes as a product $G=A B$ of subgroups $A$ and $B$ if every element of $G$ can be expressed as $a b$ where $a \in A$ and $b \in B$; if in addition, $A \cap B=\{1\}$, so that every such expression is unique, then we call this a complementary factorization. (Nearly all factorizations in this paper are complementary.) Now suppose that $G=\langle\rho, \lambda\rangle$ is the automorphism group of a regular 
Cayley map for $A$. Then since $\langle\rho\rangle$ is the stabilizer of a vertex, $G$ has a complementary factorization as $A\langle\rho\rangle$. Conversely, suppose that a group $G=\langle\rho, \lambda\rangle$, where $\lambda$ is an involution, has a complementary factorization as $A\langle\rho\rangle$. Then $A$ acts regularly on the left cosets of $\langle\rho\rangle$, and hence acts regularly on the vertices of the regular map associated with $G$. Thus we have

Proposition 2.1. The finite group A has a regular Cayley map if and only if there is a finite group $G=\langle\rho, \lambda\rangle$, where $\lambda$ is an involution, such that $G$ has a complementary factorization as $A\langle\rho\rangle$ with $A \cap\langle\rho\rangle=\{1\}$. The underlying graph of the map has multiple edges if and only if $\langle\rho\rangle$ contains a nontrivial normal subgroup of $G$.

Proof: For the last statement about multiple edges, it helps to think geometrically. Fix a vertex $v$ and an edge $e$ from $v$ to (say) $u$; let $\rho$ be the map automorphism rotating one notch about $v$, and let $\lambda$ be the map automorphism interchanging $v$ and $u$ and reversing the direction of $e$. Then $\lambda \rho \lambda$ is rotation one notch about $u$. Suppose that $e^{\prime}$ is another edge between $v$ and $u$, which is $i$ notches apart from $e$ at $v$, and $j$ notches at $u$. Then $\rho^{i}(e)=e^{\prime}$ and $\lambda \rho^{j} \lambda(e)=e^{\prime}$, so $\lambda \rho^{j} \lambda=\rho^{i}$, since the two automorphisms agree on the edge $e$. Thus $\left\langle\rho^{j}\right\rangle$ is normal in $G$. Conversely, if $\rho^{i}=\lambda \rho^{j} \lambda$, then the edge $e^{\prime}=\rho^{i}(e)=\lambda \rho^{j} \lambda(e)$ must have endpoints at $v$ and $u$, providing a second edge between $v$ and $u$.

Our interest in this paper is in regular Cayley maps without multiple edges, so we will assume throughout this paper that $\langle\rho\rangle$ contains no nontrivial normal subgroup of the map automorphism group $G$. Indeed, if $G=A\langle\rho\rangle$ is a regular Cayley map for $A$ and $N$ is the largest normal subgroup of $G$ contained in $\langle\rho\rangle$, then $G / N$ is the automorphism group of a regular Cayley map for $A$ having no multiple edges. Since we are mainly concerned with the existence of a regular Cayley map for $A$, the restriction of having no multiple edges is of no material consequence.

It is interesting to view the Platonic solids in terms of this factorization. For example, the dodecahedral map and the icosahedral map both have the alternating group $A_{5}$ as their automorphism group. Clearly, $A_{5}=\langle\rho, \lambda\rangle$ has a complementary factorization as $A_{4}\langle\rho\rangle$, where $\rho=(12345)$ and $\lambda=(12)(34)$. Since the icosahedron has valence 5, the element $\rho$ can be chosen to be the rotation about a vertex, giving a regular Cayley map for $A_{4}$. On the other hand, as the dodecahedral map has 20 vertices but $A_{5}$ has no subgroup of order 20, the dodecahedral map cannot be a regular Cayley map. The cube and octahedral maps both have as automorphism group the symmetric group $S_{4}$, which has corresponding factorizations $S_{4}=D_{4}\langle\rho\rangle$, where $D_{4}$ is dihedral of order 8 and $\rho=(123)$, and $S_{4}=S_{3}\langle\rho\rangle$ with $\rho=(1234)$. This means the standard embedding of the cube in the sphere is a regular Cayley map for $D_{4}$, and the octahedral map is a regular Cayley map for $S_{3}$. The automorphism group of the tetrahedral map is $A_{4}$, which factorizes as $V_{4}\langle\rho\rangle$ where $\rho=(123)$, making the tetrahedral map a regular Cayley map for $V_{4} \cong \mathbb{Z}_{2} \times \mathbb{Z}_{2}$.

Generally, a complementary factorization $G=A\langle\rho\rangle$ can be described simply by specifying the effect of left multiplication by $\rho$ on elements of $A$. We know for any $c$ in $A$ that $\rho c=c^{\prime} \rho^{i}$ for some unique $c^{\prime}$ in $A$ and some unique nonnegative integer $i$ less than the order of $\rho$. For each such $c$, define $f(c)=c^{\prime}$ and $p(c)=i$. Then for any $a, b$ in $A$, we have $\rho a b=f(a) \rho^{p(a)} b=f(a) \rho^{p(a)-1} f(b) \rho^{p(b)}=\cdots=f(a) f^{p(a)}(b) \rho^{k}$ for Springer 
some $k$, so $f(a b)=f(a) f^{p(a)}(b)$. A bijection $f$ of the group $A$ satisfying both this equation and $f(1)=1$ is called a skew morphism [12] for the group $A$, with associated power function $p$. Note that a skew morphism of $A$ is a group automorphism if and only if its power function is identically 1 on a generating set for $A$.

Conversely, given any skew morphism $f$ of a group $A$, we may construct the associated skew product group $G=A\langle\rho\rangle$, where $\rho$ is an element of the same order as $f$ and cross-multiplication is defined using the rule $\rho a=f(a) \rho^{p(a)}$ for every $a$ in $A$. Clearly the factorization $G=A\langle\rho\rangle$ is complementary, but in order for this group $G$ to be the automorphism group of a regular Cayley map for $A$ we need a little more. A slightly weaker version of the following proposition can be found in [18]:

Proposition 2.2. The finite group A has a regular Cayley map if and only if there is a skew morphism $f$ for $A$ and an element $x$ of $A$ such that the orbit of $x$ under $f$ generates $A$ and contains the inverse of $x$.

Proof: Suppose that $A$ has a regular Cayley map with automorphism group $G=$ $A\langle\rho\rangle=\langle\rho, \lambda\rangle$. Let $f$ be the skew morphism of $A$ defined by left multiplication by $\rho$, let $x$ be the element of $A$ such that $\lambda=x \rho^{i}$, and let $X$ be the orbit of $x$ under $f$. Clearly, $x$ and $\rho$ generate $G$ as well. The equation $\rho a=f(a) \rho^{p(a)}$, for any $a$ in $A$, allows us to rewrite any word in $x$ and $\rho$ as $w \rho^{j}$, where $w$ is a word in $X$. Thus $X$ generates $A$. Moreover, since $\lambda^{2}=1$, we have $\rho^{i} x=x^{-1} \rho^{-i}$, so $x^{-1} \in X$.

Conversely, suppose that $f$ is a skew morphism of $A$ and $x$ is an element of $A$ whose orbit $X$ generates $A$ and contains $x^{-1}$. Let $G$ be the associated skew product group $A\langle\rho\rangle$. Then $x$ and $\rho$ generate $G$, since any element $f^{i}(x)$ in $X$ can be written in $G$ as $\rho^{i} x \rho^{j}$ for some $j$. Let $\lambda=x \rho^{i}$, where $x^{-1}=f^{i}(x)$. Then $\lambda$ and $\rho$ also generate G. Moreover, $\lambda^{2}=x \rho^{i} x \rho^{i}=x x^{-1} \rho^{k}=\rho^{k}$, for some $k$, so $\rho^{k}$ commutes with all elements of $\langle\rho, \lambda\rangle=G$. Thus $a \rho^{k}=\rho^{k} a \in f^{k}(a)\langle\rho\rangle$ for all $a$ in $A$, making $f^{k}$ the identity. Since $\rho$ has the same order as $f$, we have $\lambda^{2}=\rho^{k}=1$.

This proposition is obvious if one thinks geometrically of a Cayley map with generating set $X$. If the map is also regular, the orbit of any $x$ in $X$ under the map automorphism $\rho$ rotating one notch about a vertex is all of $X$, and hence generates $A$ and contains the inverse of $x$.

The skew morphism $f$ associated with a regular Cayley map for $A$ is an automorphism of $A$ when $A$ is normal in $G=A\langle\rho\rangle$, that is, when $f$ has trivial power function, with $p(a)=1$ for all $a$ in $A$. Since $f$ is an automorphism, there are two cases:

(I) No element of $X$ is an involution, and $f^{r}(x)=x^{-1}$ for all $x$ in $X$, where $2 r$ is the valence of the map; or

(II) All elements of $X$ are involutions.

Following [21], we say that a Cayley map whose cyclic ordering of its generating set $X$ has either of these forms is balanced, of Type I or Type II, respectively. In fact:

Proposition 2.3. A regular Cayley map for the group $A$ is balanced if and only if the associated skew morphism $f$ is an automorphism. 
Proof: We have already shown that if $f$ is an automorphism, then the Cayley map is balanced. Conversely, suppose that $f$ is a skew morphism for $A$ with generating set $X$ of Type I. Then for any $y$ in $X$, we have $1=f\left(y y^{-1}\right)=f(y) f^{p(y)}\left(y^{-1}\right)=$ $f(y) f^{p(y)+r}(y)$, and then $f^{p(y)+r}(y)=f(y)^{-1}=f^{r}(f(y))=f^{r+1}(y)$. It follows that we can take $p(y)=1$ for all $y$ in $X$, and hence $f$ is an automorphism of $A$ (since $X$ generates $A$ ). Similarly, if $f$ and $X$ are of Type II, then for any $y$ in $X$, we have $1=f(y y)=f(y) f^{p(y)}(y)$, and so $f^{p(y)}(y)=f(y)^{-1}=f(y)$. Thus again $p(y)=1$ for all $y$ in $X$, and hence $f$ is an automorphism of $A$.

We say an automorphism $f$ of the group $A$ is balanced for $x \in A$ if the orbit of $x$ under $f$ generates $A$ and contains the inverse of $x$; and we say $f$ is Type II or Type I depending on whether or not $x$ is an involution. An automorphism is balanced if it is balanced for some $x$, and a group is balanced if it has a balanced automorphism.

An abelian group has a Type II balanced automorphism if and only if it is an elementary 2-group, but many nonabelian groups have such an automorphism since many are generated by involutions. The following observation has been made for simple groups also by Cai Heng Li [13], with essentially the same proof, but the argument applies to many other families of finite groups:

Theorem 2.4. Let $A=\langle x, y\rangle$ where $x$ is an involution and the normal closure of $\langle x\rangle$ is A. Then conjugation by $y$ is a Type II balanced automorphism for $x$. In particular, every nonabelian finite simple group and every symmetric group $S_{n}$ has a Type II balanced automorphism, as does any group generated by such elements where the orders of $y$ and $x y$ are relatively prime.

Proof: One needs only observe that the normal closure of $\langle x\rangle$ is generated by the conjugates of $x$ by $y$. Then use the fact $[8,14]$ that all nonabelian finite simple groups are generated by two elements, one of which is an involution. For $S_{n}$, let $x=(12)$ and $y=(12 \ldots n)$, for example. Finally in the case where the orders of $y$ and $x y$ are relatively prime, the quotient of $A$ by the normal closure of $\langle x\rangle$ is trivial.

Even though not all finite abelian groups have a balanced automorphism, all do have an automorphism with at least one orbit generating the whole group (not necessarily closed under taking inverses). Moreover, given any abelian group $A$, we can get a $\mathbb{Z}_{2}$-extension of $A$ with a Type II balanced automorphism, as follows. Define the quasidihedral group $D(A)$ by adjoining an involution $x$ such that $(x a)^{2}=1$ for all $a \in A$. The group $D\left(\mathbb{Z}_{n}\right)$ is the usual dihedral group $D_{n}$.

Proposition 2.5. Let $f$ be any automorphism of the abelian group A such that the orbit under $f$ of some element $c \in A$ generates A. Then the function $g$ defined on $D(A)$ by $g(a)=f(a)$ and $g(a x)=f(a) c x$, for all $a \in A$, is a Type II balanced automorphism for $x$.

Proof: We must verify that $g$ is indeed an automorphism of $D(A)$. We have for any $a, b \in A$ that

$$
g((a x)(b x))=g(a(x b x))=g\left(a b^{-1}\right)=f(a) f(b)^{-1},
$$


while

$$
g(a x) g(b x)=f(a) c x f(b) c x=f(a) c\left(c^{-1} f(b)^{-1}\right)=f(a) f(b)^{-1} .
$$

Similar calculations show $g((a x) b)=g(a x) g(b)$ and $g(a(b x))=g(a) g(b x)$ for $a, b \in A$. Since $g^{i}(x)=c f(c) f^{2}(c) \cdots f^{i-1}(c) x$ and the orbit of $c$ generates $A$, the orbit of $x$ generates $D(A)$.

Quasidihedral groups play a key role in later sections of this paper. Every finite abelian group $A$ has an automorphism $f$ and an element $c$ whose orbit under $f$ generates $A$. For example, if $\left\{a_{1}, a_{2}, \ldots, a_{k}\right\}$ is a canonical basis for $A$ consisting of elements $a_{i}$ of orders $m_{i}$ with $m_{1}\left|m_{2}\right| \ldots \mid m_{k}$, then let $f\left(a_{1}\right)=a_{1}$ and $f\left(a_{j}\right)=a_{j-1} a_{j}$ for $2 \leq j \leq k$, and take $c=a_{k}$. Thus every finite abelian group is a subgroup of index 2 in a group having a Type II balanced automorphism.

There are groups having a regular Cayley map, but not having a balanced one. A regular Cayley map is called anti-balanced [22] if the power function of its skew morphism has precisely the two values 1 and -1 . We will show in the next section that $A=\mathbb{Z}_{2} \times \mathbb{Z}_{4 n}$ has no balanced regular Cayley map for $n>1$, but the following result from [3] shows $A$ does have an anti-balanced one.

Theorem 2.6. There are exactly three types of anti-balanced regular Cayley maps for finite abelian groups. The following list gives abelian presentations for the groups, written additively, and partial definitions of the associated skew morphism $f$. For each type, the subgroup $B$ generated by $a$, or $a$ and $b$, has index 2 in $A$, and the power function is 1 on $B$ (so $f \uparrow_{B}$ is an automorphism) and -1 on the other coset $B+x$. For each case, the orbit of $x$ generates the group $A$ and contains the inverse of $x$.

(i) $\mathbb{Z}_{2} \times \mathbb{Z}_{2 n}=\langle a, x \mid 2 x=0,2 n a=0\rangle$, where $f(x)=a+x$ and $f(a)=a$;

(ii) $\mathbb{Z}_{2 n}=\langle a, x| 2 x=a$, na $\left.=0\right\rangle$, where $f(x)=-a+x$ and $f(a)=q a$ for some $q$ such that $q^{2} \equiv 1 \bmod n$

(iii) $\mathbb{Z}_{m} \times \mathbb{Z}_{2 m n}=\langle a, b, x| 2 x=a$, ma $=m b$, mna $\left.=0\right\rangle$, where $f(x)=-a+x$, $f(a)=b$ and $f(b)=a$.

Proof: For details, see [3]. It is an interesting exercise to check in each case that $f$ is a skew morphism, with power function 1 on $B$ and -1 on $B+x$. Note that we have only specified the effect of $f$ on $x$ and on generators for $B$; this can be extended to all of $B$ linearly and to all of $A$ by using the identity $f(c+x)=f(c)+f(x)$ for all $c$ in $B$, which must hold if $f$ is a skew morphism with power function 1 on $B$.

We remark that the underlying graph for the anti-balanced map for $\mathbb{Z}_{2 n}$ is $K_{n, n}$. In fact, any group $A$ having a subgroup of index 2 has $K_{n, n}$ as a Cayley graph (with generating set $X$ the complement of the index 2 subgroup). In the next section, we will show that any finite abelian group of odd order has a balanced regular Cayley map. Since all finite abelian groups of even order have a subgroup of index 2 , it follows that every finite abelian group has a Cayley graph that is the underlying graph of some regular Cayley map. Thus, finding regular Cayley maps for abelian groups is not really a graph-theoretic problem. 


\section{Balanced automorphisms: General groups and abelian groups of odd order}

In this section, we derive a number of results that can help determine which finite abelian groups have balanced regular Cayley maps. Some of the results of this section apply to nonabelian groups as well, and so if a group is to be abelian, we will say so.

Given an automorphism $f$ which is balanced for $x$, the smallest positive integer $r$ satisfying $f^{r}(x)=x^{-1}$ is called the semi-order of $f$. Note the following:

(1) The order of $f$ is twice its semi-order for Type I, since $f^{2 r}(x)=f^{r}\left(x^{-1}\right)=x$, and equals its semi-order for Type II, since $f^{r}(x)=x^{-1}=x$;

(2) The minimality of $r$ implies that $f^{s}(x)=x^{-1}$ only if $s$ is a multiple of $r$ and, for Type I, if and only if $s$ is an odd multiple of $r$;

(3) The rank of $A$ (the smallest number of elements required to generate $A$ ) is at most $r$, since the orbit of $x$ under $f$ generates $A$.

The following theorem applies to any finite groups $A$ and $B$, but is of particular use for abelian groups. The 2-part of the positive integer $n$ is the highest power of 2 dividing $n$.

Theorem 3.1. Let $f$ and $g$ be balanced automorphisms of $A$ and $B$ for $x$ and $y$, of semi-orders $r$ and $s$, respectively, and let $t$ be the least common multiple of $r$ and $s$. Suppose that the orders of $A$ and $B$ are relatively prime, and that $r$ and $s$ have the same 2-part. Then the automorphism $h$ of $A \times B$ defined by $h(a, b)=(f(a), g(b))$ for $(a, b)$ in $A \times B$ is balanced for $(x, y)$, and has semi-order $t$.

Proof: As $|A|$ and $|B|$ are relatively prime, the cyclic group generated by $h^{i}(x, y)=$ $\left(f^{i}(x), g^{i}(y)\right)$ contains $\left(f^{i}(x), 1\right)$ and $\left(1, g^{i}(y)\right)$. Thus the orbit of $(x, y)$ under $h$ generates $A \times B$. Since $f^{r}(x)=x^{-1}$, we have $f^{t}(x)=x^{(-1)^{m}}$ where $m=t / r$. Since $r$ and $s$ have the same 2-part, $m$ is odd, and so $f^{t}(x)=x^{-1}$. Similarly, $g^{t}(y)=y^{-1}$. Thus $h^{t}(x, y)=\left(x^{-1}, y^{-1}\right)=(x, y)^{-1}$, so $h$ is balanced for $(x, y)$, and has semiorder $t$.

The condition on the 2-parts of the semi-orders is a crucial one. We say a group $A$ is $2^{k}$-good if and only if it has a Type I balanced automorphism whose semi-order has 2-part $2^{k}$. It is important to observe that a group being $2^{k}$-good says nothing about it being $2^{j}$-good for some $j \neq k$. For example, it is not hard to see that $\mathbb{Z}_{2} \times \mathbb{Z}_{4}$ is 2 -good but not 1-good, and, as we shall see, $\mathbb{Z}_{3} \times \mathbb{Z}_{9}$ is 1 -good but not 2-good.

Theorem 3.1 says the direct product of $2^{k}$-good groups is $2^{k}$-good when the orders of the groups are relatively prime. We would like a converse.

Proposition 3.2. Let A have a balanced automorphism $f$ of semi-order $r$ for some element $x$, and let $N$ be any normal subgroup of $A$ that is invariant under $f$. Then $f$ induces a balanced automorphism of $A / N$ for $N x$ whose semi-order divides $r$ and, if $N x$ is not an involution, this semi-order has the same 2-part as $r$.

Proof: As usual, we define on the quotient group $G / N$ an automorphism, denoted again by $f$, by $f(N a)=N f(a)$. Clearly, the orbit of $N x$ under this induced 
automorphism generates $A / N$, and contains $N x^{-1}=(N x)^{-1}$. Let $s$ be the semiorder of the induced automorphism. Since $s$ is the smallest positive integer such that $f^{s}(N x)=(N x)^{-1}$, and we know $f^{r}(N x)=N x^{-1}=(N x)^{-1}$, we find that $r$ must be a multiple of $s$ and, if $N x$ is not an involution, then $r$ is an odd multiple of $s$.

Corollary 3.3. Suppose the orders of $A$ and $B$ are relatively prime. If $f$ is a balanced automorphism of semi-order $r$ for $(x, y)$ in $A \times B$, then $f$ induces balanced automorphisms $f_{A}$ on $A \times B / B \cong A$ and $f_{B}$ on $A \times B / A \cong B$ whose semi-orders divide $r$, and have the same 2-parts as $r$ if $f_{A}(x)$ and $f_{B}(y)$ are not involutions.

Proof: Since the orders of $A$ and $B$ are relatively prime, the subgroups $A_{1}=A \times\{1\}$ and $B_{1}=\{1\} \times B$ are characteristic subgroups of $A \times B$, and hence are invariant under $f$. Now apply Proposition 3.2.

As every finite abelian group $A$ is a direct product of its Sylow $p$-subgroups $A_{p}$, we have:

Corollary 3.4. Suppose A is a finite abelian group and its Sylow 2-subgroup $A_{2}$ is not elementary abelian. Then $A$ is $2^{k}$-good if and only if $A_{p}$ is $2^{k}$-good for every prime $p$.

One aim of this paper is to exhibit large classes of abelian groups that have no balanced automorphisms. It is worth observing, however, that Proposition 3.2 allows us to find also many nonabelian groups that have no balanced automorphisms:

Corollary 3.5. If the abelianization $A / A^{\prime}$ of the finite group $A$ has no balanced automorphism, then A has no balanced automorphism.

Just as a balanced automorphism of $A$ induces balanced automorphisms on certain quotients, it can also induce balanced automorphisms on certain subgroups of $A$. For any positive integer $m$, let $m A$ be the characteristic subgroup of $A$ generated by the $m$ th powers of all elements of $A$; in additive notation when $A$ is abelian, this consists of all elements of the form $m a$ for $a$ in $A$.

Proposition 3.6. Let $A$ be a finite abelian group with a balanced automorphism $f$ of semi-order $r$. Then for any positive integer $m$, the automorphism $f$ induces a balanced automorphism of $m A$ whose semi-order divides $r$, and, if $m A$ is not an elementary abelian 2-group, this semi-order has the same 2-part as $r$.

Proof: Since $m A$ is characteristic in $A, f$ restricts to an automorphism of $m A$, and we may suppose that $A$ is not an elementary abelian 2-group, since otherwise either $m A=A$ or $m A$ is trivial. Next, suppose $f$ is balanced for the element $x$ of $A$. Since the orbit of $x$ under $f$ generates $A$ and $f(m x)=m f(x)$, the orbit of $m x$ under $f$ generates $m A$, and since $f^{r}(x)=-x$ we find also $f^{r}(m x)=-m x$, so $f \uparrow_{m A}$ is a balanced automorphism of $m A$ for $m x$. Finally let $s$ be the semi-order of $f \uparrow_{m A}$. By the minimality of $s$, the semi-order $r$ of $f$ (indeed every positive integer $t$ satisfying 
$\left.f^{t}(m x)=-m x\right)$ is a multiple of $s$, and if $m A$ is not an elementary abelian 2-group, this is an odd multiple of $s$.

It is not true that a balanced automorphism of $A$ induces one on every characteristic subgroup of $A$. For example, suppose $A$ is the direct product $\mathbb{Z}_{3} \times \mathbb{Z}_{9}$, generated by commuting elements $a$ and $b$ of orders 3 and 9 , and $f$ is the balanced automorphism taking $a$ to $-a$, and $b$ to $a+2 b$; then the restriction of $f$ to the characteristic subgroup $N \cong \mathbb{Z}_{3} \times \mathbb{Z}_{3}$ generated by elements of order 3 simply takes $a$ to $-a$ and $3 b$ to $-3 b$ and is therefore not balanced, having no orbit that generates $N$. Nevertheless, and importantly, we have the following:

Theorem 3.7. If the finite abelian group $A$ is balanced, then for every characteristic subgroup $N$ of $A$, both $N$ and $A / N$ are balanced.

Proof: By Proposition 3.2, it suffices to show $N$ has a balanced automorphism. We use Pontrjagin duality, which implies that $A$ is isomorphic to the group $\operatorname{Hom}(A, \mathbb{R} / \mathbb{Z})$ of all homomorphisms from $A$ to the circle group $\mathbb{R} / \mathbb{Z} \cong S^{1}$ (see [10] for example). If $B$ is any subgroup of $A$, and $B^{*}$ is the subgroup of $\operatorname{Hom}(A, \mathbb{R} / \mathbb{Z})$ consisting of all those homomorphisms which contain $B$ in their kernel, then $A / B$ is isomorphic to $B^{*}$. The mapping $B \mapsto B^{*}$ induces a duality (an inclusion-reversing bijection) on the subgroup lattice of $A$, and this duality takes characteristic subgroups to characteristic subgroups. It follows that every characteristic subgroup of $A$ is isomorphic to a characteristic quotient of $A$, and so every characteristic subgroup of $A$ is balanced.

For the remainder of this section, we will need to describe succinctly various automorphisms of finite abelian groups. We will use additive notation, since our techniques come mostly from linear algebra. An abelian group $A$ of rank $k$ can be coordinatized by writing it in its canonical form $\mathbb{Z}_{m_{1}} \times \mathbb{Z}_{m_{2}} \times \cdots \times \mathbb{Z}_{m_{k}}$, where $m_{1}\left|m_{2}\right| \ldots \mid m_{k}$, and elements of $A$ can be added like vectors, except that entries in the $i$ th coordinate must be reduced modulo $m_{i}$ for each $i$. Any automorphism $f$ of $A$ can then be represented by a $k \times k$ matrix whose $i$ th column gives the result of applying $f$ to the element $x_{i}$ having 1 in the $i$ th coordinate and 0 elsewhere. Since $A$ is defined as the abelian group determined solely by the relations $m_{i} x_{i}=0$, to be sure that $f$ is an automorphism, we need only require that the order of the $i$ th column, regarded as an element of $A$, equals the order of $x_{i}$; this restricts the entries below the diagonal of the matrix, but not the entries above the diagonal. As usual, composition of automorphisms corresponds to matrix multiplication, with entries in the $i$ th row reduced modulo $m_{i}$.

\section{Theorem 3.8. Any finite abelian group of odd order is 1-good.}

Proof: Let $A=\mathbb{Z}_{m_{1}} \times \mathbb{Z}_{m_{2}} \times \cdots \times \mathbb{Z}_{m_{k}}$, of rank $k$, with $m_{1}\left|m_{2}\right| \ldots \mid m_{k}$, and let $m=m_{k}$, which is assumed to be odd. Let $I$ be the $k \times k$ identity matrix, and let $T$ be the $k \times k$ nilpotent matrix having 1's along the diagonal just above the main diagonal and 0 's elsewhere. Then $T^{j}=0$ for all $j \geq k$. By the binomial theorem, we have $(I+T)^{m^{k}}=I$, because for $0<j<k$ the term involving $T^{j}$ in the binomial expansion has coefficient $\left(\begin{array}{c}m^{k} \\ j\end{array}\right)$ divisible by $m$, and hence reduces to 0 modulo $m_{i}$ for 
all $i$. Since $m^{k}$ is odd, it follows that the order $s$ of $I+T$ is odd. Now let $f$ be the automorphism whose matrix is $-(I+T)$, so that $f^{s}$ is multiplication by -1 on each coordinate. It is easy to check that the orbit of $x_{k}$ under $f$ generates $A$. Thus $f$ is a balanced automorphism of $A$, of odd semi-order $s$.

Corollary 3.9. For any finite abelian group $A$, if $A_{2}$ is 1-good, then so is $A$.

Proof: Use Theorem 3.1 and Theorem 3.8.

Corollary 3.10. Every finite abelian group of rank 2 has a regular Cayley map.

Proof: Let $A=\mathbb{Z}_{m} \times \mathbb{Z}_{m n}$. If $n$ is even, then by Theorem 2.6 there exists an antibalanced regular Cayley map of type (iii) for $A$. If $n$ is odd, then the Sylow 2subgroup $A_{2}$ of $A$ is isomorphic to $\mathbb{Z}_{2^{k}} \times \mathbb{Z}_{2^{k}}$ for some $k$, and $A \cong B \times A_{2}$. It is easy to check that the rules $f(1,0)=(0,1)$ and $f(0,1)=(-1,1)$ give a balanced automorphism $f$ of semi-order 3 for $\mathbb{Z}_{2^{k}} \times \mathbb{Z}_{2^{k}}$, and so by Corollary 3.9, $A$ is 1-good (if $n$ is odd).

When $A_{2}$ is $2^{k}$-good for $k>0$, we need information about the 2-part of semi-orders of balanced automorphisms of abelian $p$-groups for odd primes $p$. To this end, write the canonical form as $A=\mathbb{Z}_{m_{1}}^{d_{1}} \times \mathbb{Z}_{m_{2}}^{d_{2}} \times \cdots \times \mathbb{Z}_{m_{s}}^{d_{s}}$, where $m_{1}<m_{2}<\cdots<m_{s}$, and $m_{1}\left|m_{2}\right| \ldots \mid m_{s}$. We then call $\mathbb{Z}_{m_{i}}^{d_{i}}$ a ledge of $A$, and the numbers $d_{1}, d_{2}, \ldots, d_{s}$ the sequence of ledge numbers for $A$. Clearly the sum of these ledge numbers is equal to the rank of $A$. Taking $m_{0}=1$, we call the numbers $r_{i}=m_{i} / m_{i-1}$ the rises of $A$. We have the following theorem:

Theorem 3.11. Let $A$ be an abelian $p$-group. If $p \equiv 1 \bmod 4$, then $A$ is 2-good. If $p \equiv 3 \bmod 4$, then $A$ is 2-good if and only if all its ledge numbers are even.

Proof: Suppose $p \equiv 1 \bmod 4$. Then for each exponent $e$, there is an integer $w$ such that $w^{2} \equiv-1 \bmod p^{e}$. Suppose that the rank of $A$ is $k$, and consider the $k \times k$ matrix $Q=S+T$, where $S$ is the diagonal matrix whose $(j, j)$ th entry satisfies $w^{2} \equiv-1$ $\bmod m_{j}$, and $T$ is the nilpotent matrix used in the proof of Theorem 3.8. Then $Q^{2}=$ $-I+T^{\prime}$, where $T^{\prime}$ is nilpotent. As in the proof of Theorem 3.8, it follows that $Q^{2 r}=$ $-I$ for some odd $r$, and that the automorphism $f$ whose matrix is $Q$ is a balanced automorphism of $A_{p}$ of semi-order $2 r$, where $r$ is odd.

Suppose that $p \equiv 3 \bmod 4$, and that $f$ is a balanced automorphism of $A$ of semiorder $2 r$, where $r$ is odd. If some ledge number is odd, then $B=p^{i} A$ has odd rank for some $i$. Letting $Q$ be the matrix for the balanced automorphism induced by $f$ on $B$, we observe that $Q$ has semi-order $2 s$, where $s$ is odd by Theorem 3.6; in particular, $Q^{2 s}=-I$, so if $w$ is the determinant of $Q^{s}$, then $w^{2} \equiv-1 \bmod p$, which is impossible for $p \equiv 3 \bmod 4$. On the other hand, suppose that every ledge number is even. We can then view all matrices for automorphisms of $A$ as being partitioned into $2 \times 2$ blocks. Let $Q$ be the matrix whose $2 \times 2$ blocks down the diagonal are all $\left(\begin{array}{lll}0 & 1 \mid-1 & 0\end{array}\right)$ and whose $2 \times 2$ blocks just above the diagonal are all $(00 \mid 10)$, and let $f$ be the automorphism of $A$ given by this matrix. It is easy to check that if $x$ is the final basis 
vector of $A_{p}$ (representing an element of maximum order in $A_{p}$ ), then the orbit of $x$ under $f$ generates $A$, and moreover, $Q^{2}=-I+T$ for some nilpotent $T$. Hence again as in Theorem 3.8, we find that $Q^{2 r}=-I$ for some odd $r$, and therefore $f$ is a balanced automorphism of $A$ of semi-order $2 r$, where $r$ is odd.

Muzychuk [16] has generalized this theorem to provide necessary and sufficient conditions for an abelian $p$-group to be $2^{k}$-good, for all odd primes $p$ :

Theorem 3.12. (Muzychuk [16]) For every odd prime $p$, if $e$ is the multiplicative order of $p$ in $\mathbb{Z}_{2^{k+1}}$, then an abelian $p$-group $A$ is $2^{k}$-good if and only if e divides all the ledge numbers of $A$. (Note that e is a power of 2.)

Proof: (Sketch; see [16] for details.) The necessity when $A=\mathbb{Z}_{p}^{n}$ follows from the action on $A$ of the cyclic subgroup of $\operatorname{Aut}(A)$ generated by a $2^{k}$-good balanced automorphism for $A$ : the point stabilizers have order divisible by $2^{k+1}$, therefore $|A-0|=p^{n}-1$ is divisible by $2^{k+1}$ and hence $e$ divides $n$. The necessity for general $A$ then follows by looking at invariant subgroups $p^{i} A$ and their quotients, using Propositions 3.2 and 3.6.

Sufficiency can be proved as in Theorem 3.11 , by constructing the appropriate matrix using $e \times e$ blocks: the matrix playing the role of the blocks down the diagonal of $Q$ corresponds to an element $w$ of the Galois field $G F\left(p^{e}\right)$ satisfying $w^{2^{k}}=-1$; such a $w$ exists since $2^{k+1}$ divides $p^{e}-1$, the order of the cyclic multiplicative group of $G F\left(p^{e}\right)$.

Regular balanced Cayley maps can be interesting even for cyclic groups. Of course, $\mathbb{Z}_{m}$ always has the trivial balanced regular Cayley map, consisting of an $m$-cycle around the equator of a sphere, corresponding to the trivial balanced automorphism $x \mapsto-x$ (the inversion mapping). We want to know which $\mathbb{Z}_{m}$ have a nontrivial balanced regular Cayley map. Perhaps it is not surprising that the characterization is in terms of elementary number theory.

Theorem 3.13. Let $m=2^{k} n>2$ where $n$ is odd. Then the cyclic group $\mathbb{Z}_{m}$ fails to have a nontrivial balanced regular Cayley map if and only if $n=1$ or $n$ is a product of distinct Fermat primes, one of which is 3 if $k=0$ or 1.

Proof: Any automorphism of $\mathbb{Z}_{m}$ is given by multiplication by an element $w$ of its multiplicative group $\mathbb{Z}_{m}^{*}$ of units, and this is a nontrivial balanced automorphism of $\mathbb{Z}_{m}$ if and only if $w^{r} \equiv-1 \bmod m$ for some $r>1$ and $w \not \equiv-1 \bmod m$.

Now if $m$ has an odd prime factor $p$ that is repeated or is not of the form $2^{s}+1$, then $\phi(m)=\left|\mathbb{Z}_{m}^{*}\right|$ has a nontrivial odd divisor (by well known properties of the Euler $\phi$-function), and it follows that $\mathbb{Z}_{m}^{*}$ has a nontrivial element $u$ of odd order, and we can let $w=-u$. Hence if $\mathbb{Z}_{m}$ has no nontrivial balanced automorphism, then $n$ has to be 1 or a product of distinct Fermat primes (each of the form $2^{s}+1$ ).

On the other hand, if $n$ is 1 or a product of distinct Fermat primes, then $\phi(m)$ is a power of 2 , so $w^{r} \equiv-1 \bmod m$ implies that $r$ is even and therefore $z^{2} \equiv-1 \bmod m$ for some $z$, in which case $z^{2} \equiv-1 \bmod 2^{k}$, which is impossible for $k>1$ (since -1 is 
not a square mod 4). Similarly if one of the primes is 3 then $w^{r} \equiv-1 \bmod m$ implies that $z^{2} \equiv-1 \bmod 3$, which is impossible. Hence in this case there is no nontrivial balanced automorphism if $k>1$ or if one of the prime factors of $n$ is 3 .

Finally, suppose that $k \leq 1$, and $n$ is a product of distinct Fermat primes, none of which is 3 . Then $2^{s-1}$ is a nontrivial square root of -1 in $\mathbb{Z}_{p}$ for each such prime $p=2^{s}+1$, and it follows from the Chinese Remainder Theorem (applied to $n$ or $2 n$ ) that there exists a nontrivial square root of -1 in $\mathbb{Z}_{m}$, and therefore also a nontrivial balanced automorphism of $\mathbb{Z}_{m}$.

Notice that if $m$ is even, we know by Theorem 2.6 that $\mathbb{Z}_{m}$ has some regular Cayley map, perhaps unbalanced. Thus the smallest example where $\mathbb{Z}_{m}$ might fail to have a nontrivial regular Cayley map of any kind is $m=15$. When we began this work some time ago, our first goal was to determine whether $\mathbb{Z}_{15}$ has an unbalanced regular Cayley map. We answer that question in Section 5.

\section{Balanced automorphisms: Abelian 2-groups}

In this section, we show that unlike the abelian groups of odd order, abelian 2-groups can fail to be balanced. First, we consider cyclic extensions of elementary abelian 2-groups, which play an important role later in the unbalanced case.

Theorem 4.1. Suppose the finite abelian group A is a cyclic extension of an elementary abelian 2-group. Then A has a regular Cayley map. Moreover, if A has rank 3 or more, then A is 1-good.

Proof: Any such group $A$ is of the form $B \times \mathbb{Z}_{2 m}$, for some $m$, where $B \cong \mathbb{Z}_{2}^{d}$ for some $d$. By Corollary 3.10, we can assume that $A$ has rank at least 3 , and $\operatorname{so} d>1$. If we view $B=\mathbb{Z}_{2}^{d}$ as the additive group of the Galois field $G F\left(2^{d}\right)$, and let $x$ correspond to a generator of the (cyclic) multiplicative group of this field, then the mapping $f$ given by $f(b)=x b$ for all $b \in B$ is an automorphism of the additive group $B$. Moreover, the orbit of $x$ under $f$ consists of all the nonzero elements of $B$. In particular, the order of $f$ is $2^{d}-1$, and the sum of all the elements in the orbit of $x$ is 0 (since exactly half of all the elements of $B$ have 1 in a given coordinate).

Viewing $A$ as $B \times \mathbb{Z}_{2 m}$, now define $g: A \rightarrow A$ by $g(b, 0)=(f(b), 0)$ for all $b$ in $B$ and $g(0,1)=(x,-1)$, with extension to all of $A$ by linearity. Note that $g(B)=B$ so $g$ is onto. Moreover, the subgroup of $A$ generated by the orbit of $(0,1)$ includes $(0,1)+(x,-1)=(x, 0)$ and hence is all of $A$. Let $n=2^{d}-1$. It is easy to verify that the $B$-coordinate of $g^{n}(0,1)$ is the alternating sum of $f^{i}(x)$ for $i=0,1, \ldots, n-1$, and since $-b=b$ for all $b$ in $B$, this is the sum of all the elements in the orbit of $x$. Thus $g^{n}(0,1)=\left(0,(-1)^{n}\right)=(0,-1)$. We conclude that $g$ is balanced for $(0,1)$, with odd semi-order $n=2^{d}-1$.

Corollary 4.2. If the Sylow 2-subgroup $A_{2}$ of the abelian group A is a cyclic extension of an elementary abelian 2-group and has rank 3 or more, then A is 1-good.

Proof: Write $A$ as $A_{2} \times B$, where $B$ has odd order and apply Theorem 3.1. 
Our main tool for constructing abelian 2-groups $A$ not having a balanced automorphism $f$ is the observation that the semi-order of $f$ cannot be less than rank of $A$. Thus we are interested in abelian groups $A$ such that the rank of $A$ is large compared to the exponent of $\operatorname{Aut}(A)$. Recall that the exponent of the group $G$ is the smallest $m$ such that $g^{m}=1$ for all $g \in G$.

Proposition 4.3. Let $N$ be a normal subgroup of the group $A$, and let $f$ be any automorphism of A under which $N$ is invariant. Let $r$ be the least common multiple of the orders of the automorphisms induced by $f$ on $N$ and $A / N$, and let $m$ be the exponent of $N$. Then the order of $f$ must divide rm.

Proof: Let $g=f^{r}$, so that $g$ induces the identity automorphism on both $N$ and $A / N$, and let $a$ be any element of $A$. Since $g$ preserves each coset of $N$, we know $g(a)=b a$ for some $b$ in $N$, and hence $g^{m}(a)=b^{m} a=a$. It follows that the order of $g$ divides $m$, and hence the order of $f$ divides $r m$.

We are mostly interested in abelian 2-groups, but the following fundamental estimate applies to all abelian $p$-groups; we expected we might find it in the literature but could not. Let $E(d, p)$ denote the exponent of $G L(d, p)$, the general linear group of $d \times d$ invertible matrices over the field with $p$ elements, where $p$ is prime.

Theorem 4.4. Let $A$ be a finite abelian p-group of exponent $p^{e}$ and largest ledge number $d$. Then the order of any automorphism of $A$ divides $E(d, p) p^{e}$. Moreover, if $A$ has only one ledge (that is, if $A$ is homocyclic), then $p^{e}$ can be replaced by $p^{e-1}$ in this upper bound.

Proof: The proof of the first assertion is by induction on $e$. When $e=1$, we have $A=\mathbb{Z}_{p}^{d}$, so any automorphism of $A$ is an element of $G L(d, p)$. The result then follows from the definition of $E(d, p)$, although there is an extra factor of $p$ that is not needed.

Now suppose that $e>1$, and that $A$ has ledge numbers $d_{1}, \ldots d_{s}$. Observe that $s \leq e$, since the $i$ th ledge has exponent at least $p^{i}$. Let $N$ be the characteristic subgroup generated by the elements of order $p$ in $A$. Define $N_{1}=N$, and for $2 \leq j \leq s$ define $N_{j}$ to be the subgroup of elements of $N$ whose coordinates are all 0 before the $j$ th ledge. Then $N_{j}=N \cap m_{j-1} A$, and hence $N_{j}$ is characteristic in $A$. Also each $N_{j}$ has exponent $p$, and $N_{j} / N_{j+1} \cong \mathbb{Z}_{p}^{d_{j}}$. Now let $f$ be any automorphism of $A$. Then $f$ leaves each $N_{j}$ invariant, and by repeated application of Proposition 4.3, starting with $N_{s}$ in $N_{s-1}$ and ending with $N_{2}$ in $N_{1}$, we find that the order of the automorphism of $f \uparrow_{N}$ divides $E(d, p) p^{s-1}$. (Note that $E(i, p)$ divides $E(i+1, p)$, since $G L(i, p)$ is isomorphic to a subgroup of $G L(i+1, p)$.) On the other hand, since $A / N$ has exponent $p^{e-1}$ and its largest ledge number is at most $d$, by induction the order of the automorphism induced by $f$ on $A / N$ divides $E(d, p) p^{e-1}$. Since $s-1 \leq e-1$, it follows that the least common multiple of the orders of automorphisms induced by $f$ on $N$ and $A / N$ divides $E(d, p) p^{e-1}$. Since $N$ has exponent $p$, by Proposition 4.3 the order of $f$ divides $E(d, p) p^{e}$.

For abelian groups with only one ledge, we begin our induction at $e=1$ but with $E(d, p)$, deleting the unnecessary extra factor $p$. The proof then proceeds as above Springer 
for $e>1$. Since $s=1$, the order of $f \uparrow_{N}$ divides $E(d, p)$, and so the least common multiple of this order and the order of $f\left\lceil_{A / N}\right.$, which by induction is now $E(d, p) p^{e-2}$, is $E(d, p) p^{e-2}$. It follows that the order of $f$ divides $E(d, p) p^{e-1}$ in this case.

If $p=2$, the bound is still too high by a factor of 2 when $A$ is cyclic. On the other hand, the bound is sharp for $A=\mathbb{Z}_{2} \times \mathbb{Z}_{4}$, for example.

Also the quantity $E(d, p)$ is not hard to compute:

Proposition 4.5. $E(d, p)=p^{e} L(d, p)$, where $p^{e} \geq d>p^{e-1}$ and $L(d, p)$ is the least common multiple of $p-1, p^{2}-1, \ldots, p^{d}-1$. In particular, $E(1,2)=1$, $E(2,2)=6$ and $E(3,2)=84$.

Proof: We sketch a proof, since we could not find this result in the literature. We note that for any $n$, viewing $\mathbb{Z}_{p}^{n}$ as the Galois field $G F\left(p^{n}\right)$ of polynomials in $x$ gives an automorphism of order $p^{n}-1$ simply by multiplying by $x$. Thus we have automorphisms of all orders dividing $L(d, p)$. We get one whose order is $p^{e}$ by using a $d \times d$ matrix whose minimal polynomial is $(x-1)^{d}$; since $(x-1)^{d}$ divides $(x-1)^{p^{e}}=x^{p^{e}}-1$, the order of the matrix divides $p^{e}$ and cannot be $p^{k}$ for $k<e$ since its minimal polynomial has degree $d>p^{e-1}$. That every automorphism $f$ has order dividing $p^{e} L(d, p)$ follows by induction on $d$, using an invariant subspace for $f$ and Proposition 4.3.

Our main interest here is 2-groups.

Theorem 4.6. Let A be a finite abelian 2-group with largest ledge number d, and suppose $f$ is a balanced automorphism for A of semi-order $r$. Then $r$ divides $2 E(d, 2)$. In particular, if A has rank greater than $2 E(d, 2)$, then A has no balanced automorphism. Moreover, if the last rise for $A$ is at least 4, then the 2-part of $r$ divides the 2-part of $E\left(d_{s}, 2\right)$, where $d_{s}$ is the final ledge number, and the odd part of $r$ divides the odd part of $E(d, 2)$. In particular, in this case, if A has rank greater than $E(d, 2)$, then A has no balanced automorphism.

Proof: Let $A$ have exponent $2^{e}$. If $e=1$, then $A=\mathbb{Z}_{2}^{d}$ and so the order and hence also the semi-order of $f$ divides $E(d, 2)$. Therefore we may suppose that $e \geq 2$. Let $N$ be the characteristic subgroup $2^{e-2} A$. If the last rise is 2 , then $A / N \cong \mathbb{Z}_{2}^{d_{s-1}} \times \mathbb{Z}_{4}^{d_{s}}$, so the order of the automorphism induced by $f$ on $A / N$ divides $E(d, 2) 2^{2}$, and hence its semi-order $r^{\prime}$ divides $2 E(d, 2)$. If the last rise is at least 4 , then we can apply the oneledge case of Theorem 4.4 to eliminate a factor of 2 , and we can obtain a bound for the 2-part of $r^{\prime}$ by simply using the last ledge number $d_{s}$ instead of $d$. By Proposition 3.2, the semi-order $r$ of $f$ must have the same 2-part as $r^{\prime}$, and the odd part of $r$ must divide $E(d, 2)$. Thus in all cases, $r$ also divides $2 E(d, 2)$, and if the last rise is at least 4 , then the 2-part of $r$ divides $E\left(d_{s}, 2\right)$. The remarks about the rank of $A$ and the nonexistence of a balanced automorphism for $A$ follow from the observation that if a group has a balanced automorphism of semi-order $r$, then the rank of $A$ is at most $r$. 
Corollary 4.7. Let $A$ be an abelian 2-group with largest ledge number 1 . If A has a balanced automorphism of semi-order $r$, then either $r=1$ and $A \cong \mathbb{Z}_{n}$, or $r=2$ and $A \cong \mathbb{Z}_{n} \times \mathbb{Z}_{2 n}$, for some $n>1$.

Proof: By Theorem 4.6, the rank of $A$ is at most $2 E(1,2)=2$. We already know $\mathbb{Z}_{2^{k}}$ has only the trivial balanced automorphism. For rank 2, again by Theorem 4.6 the last rise must be 2 . If we write $\mathbb{Z}_{n} \times \mathbb{Z}_{2 n}$ as $\langle a, b \mid(2 n) a=0, n a=n b\rangle$, then the automorphism $f$ given by $f(a)=b$ and $f(b)=-a$ is balanced, of semi-order 2 , and is clearly unique up to a change of basis.

The necessary condition that the rank of $A$ be at most $2 E(d, 2)$ is by no means sufficient to guarantee a balanced automorphism. For one thing, we have observed in Corollary 4.7 that not all groups of rank 2 have a balanced automorphism. A more interesting example is $A=\mathbb{Z}_{2} \times \mathbb{Z}_{4} \times \mathbb{Z}_{4} \times \mathbb{Z}_{8}$. The last rise for this group is 2, and its rank is 4 , which certainly divides $2 E(d, 2)=12$. By computer calculations however, using the MAGMA system [2], we know that $A$ is not balanced, although it does have a regular Cayley map.

Muzychuk [16] has obtained necessary and sufficient conditions that an abelian 2 -group be $2^{k}$-good. The conditions are too technical to state here succinctly, except for the 1-good case:

Theorem 4.8. (Muzychuk) Let $N_{i}$ be the number of irreducible polynomials of degree $i$ over $G F(2)$, for $i \geq 2$, and let $N_{1}=1$. Then a finite abelian 2-group $A$ is 1-good if and only if it is a direct sum of abelian groups of the form $\left(A_{i}\right)^{i}$ where $A_{i}$ has rank at most $N_{i}$.

This theorem provides a necessary condition that is easy to check:

Corollary 4.9. Suppose the abelian 2-group $A$ is 1-good. Let $L_{i}$ be the number of ledges of length $i$ for $A$. Then $\sum_{i=1}^{n} i L_{i} \leq \sum_{i=1}^{n} i N_{i}$ for all positive integers $n$.

The condition is not sufficient: if $L_{4}=5$ and all other $L_{i}=0$, then $A$ is not 1good, but the necessary inequality is satisfied for all $n$ since $N_{2}=1, N_{3}=2, N_{4}=3$. In general, Muzychuk's characterization for 1-good 2-groups depends on the existence of restricted partitions of multisets, and it is not clear whether there is a computationally efficient way of determining this.

\section{Ito's theorem and the $B C D$ structure of the automorphism group}

To understand unbalanced regular Cayley maps, we must analyze the structure of the automorphism group $G$. Since we will be dealing with multiplication in the nonabelian group $G$, we will no longer use additive notation for abelian groups. Also, to emphasize the algebraic nature of our proofs, we will write $y$ instead of $\rho$, and $x$ instead of $\lambda$, so that $x$ and $y$ generate $G$, and $G=A Y$ where $Y=\langle y\rangle$ and $A \cap Y=\{1\}$.

For the rest of this paper, we let $C$ denote the commutator subgroup $G^{\prime}$ and $D$ be the normal closure of $x$, that is, the smallest normal subgroup of $G$ containing $x$. Because $G / D$ is cyclic (generated by the coset $D y$ ), we know $C$ is contained in $D$. Moreover, Springer 
as $G / D$ can be obtained from the abelian group $G / C$ by making trivial the coset of the involution $x$, the subgroup $C$ has index at most 2 in $D$. We also let $f$ denote the automorphism of $D$ given by conjugation by $y$, and we let $s$ denote the order of $y$. The following Proposition applies to all finite groups.

Proposition 5.1. The automorphism $f$ given by conjugation by y is a Type II balanced automorphism of $D$ for $x$. If $C$ has index 2 in $D$, then $f(x)=c x$ for some $c$ in $C$, where the orbit of $c$ under $f$ generates $C$, and conjugation by $x$ sends each of the elements $c, f(c) c, f^{2}(c) f(c) c, \ldots, f^{s-1}(c) f^{s-2}(c) \ldots f^{2}(c) f(c) c$ to its inverse.

Proof: Since $D$ is generated by the conjugates of $x$ by powers of $y$, the orbit of $x$ under $f$ generates $D$. Since $x$ is an involution, this makes $f$ a Type II balanced automorphism of $D$.

Now suppose that $C$ has index 2 in $D$. Since the commutator subgroup is characteristic, $f$ leaves $C$ invariant, and so $f(x)=c x$ for some $c$ in $C$. It then follows that $f^{2}(x)=f(c) c x, f^{3}(x)=f^{2}(c) f(c) c x, \ldots$, and so on. Since $f$ is an automorphism and $x$ is an involution, the element $f^{i}(x)$ is also an involution for each $i$. Hence for each element $w$ in the set $S=\left\{c, f(c) c, f^{2}(c) f(c) c, \ldots, f^{s-1}(c) f^{s-2}(c) \ldots f^{2}(c) f(c) c\right\}$ (of elements $f^{j}(x) x$ for $1 \leq j \leq s$ ), we have $(w x)^{2}=1$, and therefore the involution $x$ conjugates $w$ to its inverse. In particular, $x$ normalizes the subgroup generated by this set $S$, and so this subgroup has index 2 in the subgroup $D$ (generated by the $f$-orbit of $x$ ). But also each element of $S$ lies in $C$ (since $C$ is invariant under $f$ ), so $S$ generates $C$, and thus the $f$-orbit of $c$ generates $C$.

Of course we are mainly interested in the case when $A$ is abelian. In this case, we can use Ito's theorem to understand the structure of $G=A Y$ :

Theorem 5.2. (Ito [11]) Let $G=A Y$ where $A$ and $Y$ are abelian subgroups of $G$. Then the commutator subgroup $G^{\prime}$ is abelian.

Theorem 5.3. Let $G=A Y$ be the automorphism group of a regular Cayley map for the finite abelian group $A$, where $Y$ is cyclic of order $s$. Then $C=G^{\prime}$ is abelian, and conjugation by $x$ inverts all elements of $C$. Moreover, there are three possibilities:

(1) $C=D$ is an elementary abelian 2-group (necessarily of the same order as $A$ ), $G / C=G / D \cong \mathbb{Z}_{s}$, and $G=C Y=D Y$ with $C \cap Y=D \cap Y=\{1\} ;$

(2) $C \neq D, G / C \cong \mathbb{Z}_{s}, G / D \cong \mathbb{Z}_{s / 2}$ (with $s$ even and $x y^{s / 2} \in C$ ), and $G=C Y$ with $C \cap Y=\{1\}$

(3) $C \neq D, G / C \cong \mathbb{Z}_{s} \times \mathbb{Z}_{2}, G / D \cong \mathbb{Z}_{s}$, and $G=D Y$ with $D \cap Y=\{1\}$.

In the last two cases, $D$ is the quasidihedral group $D(C)$.

Proof: By Ito's Theorem, $C$ is abelian. By Proposition 5.1, conjugation by $x$ inverts all elements of a generating set for $C$, and hence inverts all elements of $C$. In particular, if $y^{i}$ is in $C$, then $x y^{i} x=y^{-i}$, so $\left\langle y^{i}\right\rangle$ is normal in $G$, contradicting our assumption about no multiple edges. Thus $C \cap Y=\{1\}$, and so $G / C \cong \mathbb{Z}_{s}$, generated by $C y$, or $G / C \cong \mathbb{Z}_{s} \times \mathbb{Z}_{2}$, generated by $C y$ and $C x$. If $G / C \cong \mathbb{Z}_{s}$, then $G=C Y$ and we have case (1) if $C=D$, or case (2) if $C \neq D$. Also if $C=D$, then since $D$ is generated 
by involutions (namely the conjugates of $x$ ), we find that $C$ is an elementary 2-group. On the other hand, if $G / C \cong \mathbb{Z}_{S} \times \mathbb{Z}_{2}$, then since $x$ is not in $C$ we know $C \neq D$, and then since $G / D \cong(G / C) /(D / C) \cong \mathbb{Z}_{s}$, we have $D \cap Y=\{1\}$ and $G=D Y$.

In the previous section, we discussed the particular case of cyclic groups and the $\mathbb{Z}_{15}$ problem that motivated us. Using Theorem 5.3, we can now determine exactly which cyclic groups fail to have a nontrivial regular Cayley map.

Corollary 5.4. The only finite cyclic groups failing to have a nontrivial regular Cayley map are those of order a product of distinct Fermat primes one of which is 3.

Proof: We already know by Theorem 2.6 that if $n$ is even, then $\mathbb{Z}_{n}$ has an antibalanced regular Cayley map, and if $n$ is odd and not a product of distinct Fermat primes one of which is 3, then $n$ has a balanced regular Cayley map. We assume therefore that $n$ is a product of distinct Fermat primes, one of which is 3, and that $G=A Y$ is the automorphism group for a regular Cayley map for $A=\mathbb{Z}_{n}$. Case (3) of the previous theorem cannot hold, since in that case $|D|$ is even yet also $|D|=|A|=n$. Thus $G=C Y$, where $C$ is abelian, and $|C|=|A|=n$. Since $n$ is square-free, every abelian group of order $n$ is cyclic, and so $C \cong \mathbb{Z}_{n}$. Since $C$ is normal in $G$, we have a balanced regular Cayley map for $\mathbb{Z}_{n}$, contradicting Theorem 3.13.

The last ingredient we require for considering the structure of $A$ and $G$ is the subgroup $B=A \cap C$. The following proof that $B$ is normal in $G$ is a modification of an argument by Muzychuk [16].

Proposition 5.5. B is normal in $G$.

Proof: First observe that if $g=a y^{i}$ commutes with all elements of $A$, then so does $y^{i}$, making $\left\langle y^{i}\right\rangle$ normal in $G$, contradicting our assumption in this paper that $Y$ is core-free, unless $i=0$, in which case $g=a \in A$. Next, since $C$ is normal in $G$ and conjugation by $x$ inverts all elements of $C$, to prove $B=A \cap C$ is normal in $G$ it suffices to show for a given $b \in B$ that $y b y^{-1} \in A$. For any $a \in A$, the element $y^{-1} a y a^{-1} \in C$ commutes with $b$ since $C$ is abelian. Then since $a^{-1}$ commutes with $b$, also $y^{-1} a y$ commutes with $b$, so $y^{-1} a y b=b y^{-1} a y$, which can be rewritten as $a y b y^{-1}=y b y^{-1} a$. Thus $y b y^{-1}$ commutes with all elements of $A$, so by our first remark, $y b y^{-1} \in A$.

Next, we show that $A$ is a cyclic extension of $B$, for the cases that interest us.

Theorem 5.6. In cases (1) and (2) of Theorem 5.3, the group A is a cyclic extension of $B$. In case (3), either $A$ is a cyclic extension of $B$, or $A$ is a cyclic extension of an elementary abelian 2-group.

Proof: We note that $A / B=A /(A \cap C) \cong A C / C$ is isomorphic to the image of $A$ in the quotient group $G / C$. In cases (1) and (2), we know $G / C$ is cyclic, so $A / B$ is cyclic. In case (3), we know that $G / D$ is cyclic, so $A /(A \cap D) \cong A D / D$ is cyclic. Also $|D / C|=2$, so $B=A \cap C=A \cap D \cap C$ has index 1 or 2 in $A \cap D$. We claim 祭 Springer 
that either $B=A \cap D$, making $A / B$ cyclic, or otherwise $A \cap D$ is an elementary abelian 2-group. If $B \neq A \cap D$, then $A \cap D$ can be generated by $B$ together with one element of the form $c x$ for some $c$ in $C$. This element is an involution, since $x c x=c^{-1}$, and commutes with every element of $B$ (since $A \cap D \subseteq A$ is abelian). But also if $b$ is in $B$, then $(c x) b(c x)^{-1}=c x b x\left(c^{-1}\right)=c b^{-1} c^{-1}=b^{-1}$, so $b=b^{-1}$, and thus $b^{2}=1$ for all $b$ in $B$. We conclude that $A \cap D$ is an elementary abelian 2-group.

Next, we would like to eliminate cases (1) and (2) of Theorem 5.3 from consideration, for the abelian groups that interest us.

Theorem 5.7. If case (1) occurs, or case (2) occurs and $|A / B|$ is even, then $A$ is a cyclic extension of an elementary abelian 2-group. In particular, if $A$ is an abelian 2-group that is not a cyclic extension of an elementary abelian 2-group, then either $A=C$ and $f$ is a balanced automorphism of $A$, or case (3) occurs.

Proof: If case (1) occurs, then $C$ itself is an elementary abelian 2-group, and therefore so is $B=A \cap C$. Suppose now that case (2) occurs and $|A / B|=m$ is even. Since $A / B$ is cyclic, $A$ can be obtained from $B$ by adjoining an element $c y^{i}$, for some $c$ in $C$ and some $i$, such that $\left(c y^{i}\right)^{m}$ is in $B$. Since $C$ is normal, $\left(c y^{i}\right)^{j}=c^{\prime} y^{i j}$ for some $c^{\prime}$ in $C$, and so when we consider the images in $Y \cong G / C$ of the elements $c y^{i},\left(c y^{i}\right)^{2},\left(c y^{i}\right)^{3}, \ldots,\left(c y^{i}\right)^{m}$, we should see the cyclic subgroup of $Y \cong \mathbb{Z}_{s}$ of order $m$ generated by $y^{i}$. Since $m$ is even, one of these images will be $y^{s / 2}$, and thus $c^{\prime} y^{s / 2}$ is in $A$ for some $c^{\prime}$ in $C$. The element $c^{\prime}$ commutes with every element of $B$ since $C$ is abelian, and $c^{\prime} y^{s / 2}$ commutes with every element of $B$ since $A$ is abelian, so $y^{s / 2}$ also commutes with every element of $B$. But also in case (2), since $C$ is normal in $G$ we know that conjugation by $y$ induces a balanced automorphism of $C$, of semi-order $s / 2$, and so conjugation by $y^{s / 2}$ takes $b$ to $b^{-1}$ for all $b$ in $B$. Thus $b=b^{-1}$ for all $b$ in $B$, and therefore $A$ is a cyclic extension of the elementary abelian 2-group $B=A \cap C$.

Finally suppose $A$ is a finite abelian 2-group that is not a cyclic extension of an elementary abelian 2-group. If $|A / B|=1$, then $A=B=C$, so $f$ is a balanced automorphism of $A$. On the other hand, if $|A / B|>1$, then $|A / B|$ is even, and cases (1) and (2) are ruled out by the above argument, so case (3) must occur.

Theorem 5.8. Suppose that case (3) occurs, but A is not a cyclic extension of an elementary abelian 2-group, and that $A / B \cong \mathbb{Z}_{m}$. Then $A$ can be obtained from the subgroup $B$ by adjoining an element of the form $d z$, where $z=y^{r}, r=s / m$, and $d$ is an element of $D$. Moreover, $(d z)^{i}=d_{i} z^{i}$ for $0 \leq i<m$, where the $d_{i}$ form a complete system of distinct coset representatives for $B$ in $D$. In particular, $d=d_{1}=c x$ for some $c$ in $C$, conjugation by $z=y^{r}$ inverts all elements of $B$, and $r$ is the smallest positive integer $j$ such that $y^{j}$ has this property.

Proof: We know $A$ is a cyclic extension of $B$, so it can be obtained from $B$ by adjoining some element of $G=D Y$. Moreover, since $A \cap D=B$, this element is not in $D$ and so must be of the form $d z$, for some $d$ in $D$ and $z=y^{r}$ where $0<r<s$. Since $D$ is normal, $(d z)^{j}=d^{\prime} z^{j}$ for some $d^{\prime}$ in $D$, and as in the previous proof, $z$ generates a cyclic subgroup of order $m$ in $\langle y\rangle \cong \mathbb{Z}_{s}$. Thus we can take $r=s / m$. Now define $d_{i}$ by $(d z)^{i}=d_{i} z^{i}$ for $0 \leq i<m$. Suppose that $d_{j}=b d_{i}$, for $j \neq i$ and some $b$ in $B$. 
Then $(d z)^{j-i}=(d z)^{-i}\left(b d_{i} z^{j}\right)=b(d z)^{-i}\left(d_{i} z^{j}\right)$, since $d z$ commutes with $b$ (both being elements of $A$ ). Thus $(d z)^{j-i}=b z^{-i} d_{i}^{-1} d_{i} z^{j}=b z^{j-i}$. Since both $d z$ and $b$ are in $A$, this means $z^{j-i}$ is in $A$, contradicting the factorization $G=A Y$.

It follows that the $d_{i}$ make up a set of $m$ distinct coset representatives of $B$ in $D$, and as $|D / B|=|A / B|=m$, this is a complete set. In particular, not all of the $d_{i}$ can be in $C$. If $d=d_{1}$ were in $C$, then all the $d_{i}$ would be in $C$ since $z$ normalizes $C$, and therefore $d=c x$ for some $c$ in $C$. Since $c$ commutes with all elements of $C$ and conjugation by $x$ inverts all elements of $c$, conjugation by $d$ inverts all elements of $C$. Since $d z$ commutes with $B$, this means conjugation by $z=y^{r}$ must also invert all elements of $B$.

Now let $j$ be the smallest positive integer $j$ such that conjugation by $w=y^{j}$ inverts all elements of $B$. Since conjugation by $x$ also inverts all elements of $B$, the subgroup $A^{\prime}$ of $G$ generated by $B$ and $x w$ is abelian. If $j<r$, then the set of all powers of $x w$ has more than $m$ elements. If we let $(x w)^{i}=e_{i} w^{i}$ where $e_{i}$ is in $D$ for each $i$, then two of the $e_{i}$ must lie in the same coset of $B$ in $D$, say $e_{j}=b e_{i}$, and then, as before, we can argue that $(x w)^{j-i}=b w^{j-i}$, which implies that $w^{j-i}$ is in the abelian group $A^{\prime}$. Then $w^{j-i}$ commutes with $x w$, so $w^{j-i}$ commutes with $x$. This implies, however, that the subgroup of $Y=\langle y\rangle$ generated by $w^{j-i}$ is normal in $G$, a contradiction. Hence $j=r$, and $w=y^{r}=z$.

Our final goal is to show that $C$ is a cyclic extension of $B$, and isomorphic to a subgroup of index at most 2 in $A$. We will need the following recent result of Conder and Isaacs [5], which was motivated by the investigations carried out for this paper:

Theorem 5.9. If the finite group $G$ is expressible as a product $X Y$ of an abelian subgroup $X$ and a cyclic subgroup $Y$, then $G^{\prime} /\left(G^{\prime} \cap X\right)$ is cyclic and isomorphic to a subgroup of $Y /(X \cap Y)$, and $G^{\prime} /\left(G^{\prime} \cap Y\right)$ has rank no more than that of $X$ and is isomorphic to a subgroup of $X /(X \cap Y)$.

As a consequence we have the following:

Theorem 5.10. If $G=A Y$ is the automorphism group of a regular Cayley map for the finite abelian group $A$, then $C / B=G^{\prime} /\left(G^{\prime} \cap A\right)$ is cyclic, and $C=G^{\prime}$ is isomorphic to a subgroup of $A$.

Proof: In this case (with $X=A$ ) we have $X \cap Y=\{1\}$ and also $G^{\prime} \cap Y=\{1\}$.

We originally had a self-contained proof of this theorem, but it seems best to place this result in the larger context of the theorem of Conder and Isaacs. We should note, however, that our original proof contained a little more information in case (3) when $A$ is not a cyclic extension of an elementary 2-group: there are elements $u$ in $A$ and $v$ in $C$ such that $v^{m / 2}=u^{m}$, where $A / B \cong \mathbb{Z}_{m}$. This allows, for example, a shorter proof of the fact that $\mathbb{Z}_{2} \times \mathbb{Z}_{4} \times \mathbb{Z}_{8}$ has no regular Cayley map-see later.

Theorem 5.11. For every odd prime $p$, the Sylow $p$-subgroups $A_{p}$ and $C_{p}$ are isomorphic, and either $A_{2} \cong C_{2}$ or otherwise $A_{2}$ is isomorphic to a $\mathbb{Z}_{2}$-extension of $C_{2}$. 
In particular, $C$ is isomorphic to $A$ in cases (1) and (2), while $C$ is isomorphic to an index 2 subgroup of $A$ in case (3).

Proof: Compare orders of the subgroups $A$ and $C$ in each case.

Theorem 5.12. If the abelian group $A$ is not balanced but has a regular Cayley map, then it has an index 2 subgroup $C$ that is a cyclic extension of a balanced group $F$.

Proof: Since $A$ is not balanced, a regular Cayley map for $A$ must be one from case (3). Let $B, C, D, x, y, c, f$ be as in Proposition 5.1. Since $C$ is a cyclic extension of $B$, $f(c)=b c^{q}$, where $q$ is a unit in $\mathbb{Z}_{m / 2} \cong C / B$. Thus $c$ together with the orbit of $b$ under $f$ generate $C$. If $F$ is the subgroup of $B$ generated by the orbit of $b$, then $C$ is a cyclic extension of $F$. Then by Theorem 5.8, $f$ is a balanced automorphism of $F$ of semi-order $r=s / m$.

\section{Abelian groups having no regular Cayley maps}

In this section we construct infinitely many finite abelian groups that have no regular Cayley map at all. We need first a lemma showing that the largest ledge number changes by at most one when taking cyclic extensions.

Lemma 6.1. Suppose the finite abelian group $A$ is a cyclic extension of the abelian group $B$. Then the largest ledge number for $B$ is at most one more than the largest ledge number of $A$.

Proof: We revert here to additive notation for abelian groups. Suppose that $A$ can be obtained from $B$ by adjoining an element $a$ such that $n a=b$ for some $b$ in $B$, where $n$ is the index of $B$ in $A$. Let $\mathbb{Z}_{m}^{d}$ be any ledge of $B$, and write the coordinates of $b$ with respect to some basis of this ledge as $s\left(k_{1}, k_{2}, \ldots, k_{d}\right)$, where $0 \leq k_{i}<m$ for each $i$, and $\operatorname{gcd}\left(k_{1}, k_{2}, \ldots, k_{d}\right)=1$. Then there exists a basis for $\mathbb{Z}_{m}^{d}$, as a module over $\mathbb{Z}_{m}$, whose first basis vector is $\left(k_{1}, k_{2}, \ldots, k_{d}\right)$, and with respect to this $d$-element basis for the ledge under consideration, the coordinates of $b$ become $(s, 0, \ldots, 0)$. It follows that in the group $A$, the elements of this ledge for $B$ whose (new) first coordinate is 0 comprise part of a ledge of $A$ of length at least $d-1$. In particular, the largest ledge number of $B$ is at most one more than the largest ledge number of $A$.

Theorem 6.2. Let $A$ be a finite abelian 2-group with largest ledge number d and rank greater than $2 E(d+2,2)+2$. Then A has no regular Cayley map.

Proof: Suppose instead that $A$ does have a regular Cayley map, with automorphism group $G=A\langle y\rangle$. Then we know that $A$ is not a cyclic extension of $\mathbb{Z}_{2}^{d}$, since otherwise its rank is at most $d+1$, which is clearly less than $2 E(d+2,2)$. Also by Theorem 4.6, we know that $A$ has no balanced automorphism so we can use Theorem 5.12. Next, we know that $C$ is a cyclic extension of a balanced group $F$, so the rank of $A$ is at most 2 more than the rank of $F$. By Lemma 6.1, we know the largest ledge number 
for $F$ is at most $d+2$. Thus the rank of $F$ is greater than $2 E(d+2,2)$, contradicting Theorem 4.6.

For example, Theorem 6.2 says that if $A$ has largest ledge number 1 and rank greater than $2 E(3,2)+2=170$, then $A$ has no regular Cayley map. Clearly this rank bound is excessive: for example, the odd-part of the semi-order of $f$ divides $E(d+1,2)$, since $f$ is an automorphism of $C$, which has largest ledge number $d+1$. In addition, if all the rises of $A$ are greater than 2, then $C$ has the same largest ledge number as $A$, and the rank bound reduces further:

Theorem 6.3. Let $A$ be a finite abelian 2-group with largest ledge number 1 and all rises at least 4 . If the rank of $A$ is 6 or more, or if the last two rises of $A$ are at least 8 and the rank of $A$ is 4 or more, then A has no regular Cayley map.

Proof: Assume that this group $A$ does have a regular Cayley map. Since the rises of $A$ are all at least 4, the largest ledge numbers of $A$ and $C$ are both 1 and the ranks of $A$ and $C$ are the same. Let $r$ be the semi-order of $f \uparrow_{F}$. The odd part of $r$ is 1 (since $f \uparrow_{C}$ has order a power of 2), and the 2-part of $r$ divides the 2-part of $2 E(2,2)=12$. Hence the rank of $F$ is at most 4, and so the ranks of $C$ and $A$ are at most 5 .

If the last two rises of $A$ are at least 8, then the last two rises of $C$ are both at least 4. As in the proof of Theorem 4.6 using last ledge number 1 or 2 , we find that $\mathbb{Z}_{4}, \mathbb{Z}_{2} \times \mathbb{Z}_{4}$ or $\mathbb{Z}_{4} \times \mathbb{Z}_{4}$ is a characteristic quotient of $F$. This saves an extra factor of 2 , since now the 2-part of the order of $f \uparrow_{F}$ must divide the 2-part of $E(2,2) 2^{2-1}=12$, so the semi-order $r$ divides 2 . Thus the rank of $F$ is at most 2, and the ranks of $C$ and $A$ are at most 3 .

To extend these results to groups other than 2-groups, we have

Theorem 6.4. Let $A$ be any finite abelian group. If $A_{2}$ has no regular Cayley map, then neither does $A$.

Proof: Suppose $G=A Y$ and let $C=G^{\prime}$ as usual. Let $H$ be the Hall 2-complement (the product of the Sylow subgroups of odd order) of $C$. Then $H$ is characteristic in $C$ and hence also in $G$. Let $\eta: G \rightarrow G / H$ be the natural homomorphism. Since $A_{2} \cap H=\{1\}$ and $Y \cap H=\{1\}$, we know that $\eta\left(A_{2}\right) \cong A_{2}$ and $\eta(Y) \cong Y$. We claim that $G / H$ has a complementary factorization as $\eta\left(A_{2}\right) \eta(Y)$, which then makes $G / H$ the desired automorphism group of a regular Cayley map for $\eta\left(A_{2}\right)$, a contradiction. Since $|A|=|C|$ or $2|C|$, we know the Hall 2-complement of $A$ has the same order as $H$, so $|G / H|=\left|A_{2}\right||Y|$, and therefore it suffices to show that $G / H=\eta\left(A_{2}\right) \eta(Y)=$ $\left(A_{2} H / H\right)(H Y / H)=A_{2} H Y / H$. This in turn follows if we can show that $G=A_{2} H Y$, and as $|G|=\left|A_{2}\right||H||Y|=\left|A_{2}\right||H Y|$, it actually suffices to show that $A_{2} \cap H Y=$ $\{1\}$.

Since $|H|$ is odd, the unique (cyclic) Sylow 2-subgroup of $Y$ is a Sylow 2-subgroup of $H Y$, and so any element of $H Y$ whose order is a power of 2 is conjugate to an element of $Y$. In particular, if $a_{2}$ is in $A_{2} \cap H Y$, then $g^{-1} a_{2} g=y^{i}$ for some $g$ in $G=A Y$, say $g=a y^{j}$ where $a$ is $A$; but then $a_{2}=a^{-1} a_{2} a=a^{-1} g y^{i} g^{-1} a=y^{j} y^{i} y^{-j}=y^{i}$, and 祭Springer 
as $A \cap Y=\{1\}$ this implies $a_{2}=1$. Hence $A_{2} \cap H Y=\{1\}$, and therefore $G / H=$ $\eta\left(A_{2}\right) \eta(Y)$ as claimed.

When we first began this study, our goal was to find just one finite abelian group having no regular Cayley map. The first we found was $A=\mathbb{Z}_{2} \times \mathbb{Z}_{4} \times \mathbb{Z}_{8}$; this, however, was achieved with the help of an exhaustive computer search. Although this now follows from the recent work of Muzychuk [17], we give a proof to indicate how the $B C D$ structure can be used.

Theorem 6.5. The group $A=\mathbb{Z}_{2} \times \mathbb{Z}_{4} \times \mathbb{Z}_{8}$ has no regular Cayley map.

Proof: Assume that $A$ does have a regular Cayley map, with automorphism group $G=A Y$ as usual. Since $A$ has no balanced automorphism, the map must be one from case (3). Let $B, C, D, x, c, r, m, s$ be as in Theorem 5.8 and let $f, b, F$ be as in Theorem 5.12. To shorten the proof, we will assume that $A$ and $C$ are obtained from $B$ by adjoining elements $u$ and $v$, respectively, such that $v^{m / 2}=u^{m}$.

The proof is by cases for $m$. If $m=2$, then $B=C$ so $A$ contains the commutator $C$ making $A$ normal in $G$, a contradiction. Since $A$ has exponent 8 , the only cases are $m=4$ or 8 . For notational convenience, we will abbreviate abelian groups simply by listing their canonical factors, so that $A=[2,4,8]$ for example. We also list an element by its canonical coordinates, for example $(1,3,2)$ stands for $a_{1}^{1} a_{2}^{3} a_{3}^{2}$ where $a_{1}, a_{2}, a_{3}$ are canonical generators for [2, 4, 8].

The case $m=4$ : The possibilities, up to a change in canonical basis for $B$, are $B=$ $[2,8]$ and $u^{4}=(0,0), B=[2,2,4]$ and $u^{4}=(0,1,0)$ (since $u^{4}=(0,0,2)$ would give $A=[2,2,2,8]), B=[4,4]$ and $u^{4}=(2,0)$. In the first case, since $C$ is obtained by adjoining $v$ with $v^{2}=u^{4}$, we have $C=[2,2,8]$, and in the other two cases we have $C=[2,4,4]$. In particular, $F$ must have rank at least 2 since $C$ has rank 3, so $r>1$.

Assume that $r$ is even. Since $B$ has index 2 in $C$, any product of an even number of elements not in $B$ is in $B$, and hence $f^{r}(x)=f^{r-1}(c) f^{r-2}(c) \ldots f^{2}(c) f(c) c x=b^{\prime} x$ for some $b^{\prime}$ in $B$. It follows that $f^{2 r}(x)=f^{r}(b x)=f^{r}(b) b x=b^{-1} b x=x$, so $f$ has order $2 r$, a contradiction since $s=m r=4 r$. Thus $r>1$ is odd. This means the case $B=[2,8]$ is impossible, since $[2,8]$ has no automorphism of odd order. Moreover, in all the cases, $r$ must divide the odd part of $E(2,2)=6$, so $r=3$.

Suppose that $B \cong[2,2,4]$. Let $N$ be the subgroup of $C$ generated by the involutions in $C$, which is characteristic in $C$. Then $2 B \subset 2 C \subset N \subset B$, with all containments strict and subgroups invariant under $f$. By repeated application of Proposition 4.3, it follows that $f \uparrow_{B}$ has order a power of 2, contradicting $r=3$.

Suppose instead that $B \cong[4,4]$. Let $b^{\prime}=c f^{3}(c)$. Since $B$ has index 2 in $C, b^{\prime} \in B$. Moreover, $\left(b^{\prime}\right)^{2}=c^{2} f^{3}\left(c^{2}\right)=1$, since $c^{2} \in B$. Then $f^{6}(x)=f^{2}\left(b^{\prime}\right) f\left(b^{\prime}\right) b^{\prime} x$. Let $N$ be the characteristic subgroup of $B$ generated by involutions in $B$. Then $N \cong[2,2]$ and $b^{\prime} \in N$. Since $f^{3}$ inverts elements of $B, f^{3}$ is the identity on $N$. Since $c$ and $f(c)$ generate $B$, we must have $f\left(c^{2}\right) \neq c^{2}$, so $f \uparrow_{N}$ has order 3 . Then the orbit of $b^{\prime}$ under $f$ consists of all three nonidentity elements of $N$, so $f^{2}\left(b^{\prime}\right) f\left(b^{\prime}\right) b^{\prime}=1$. Therefore $f^{6}(x)=x$, contradicting $s=12$.

The case $m=8$ : Here $B$ has index 4 in $C$, and $f$ has order $8 r$. Since $A$ has exponent $8, u^{8}=v^{4}=1$, so $B \cong[2,4]$ and $C \cong[2,4,4]$. Again, since $C$ has rank $3, F$ must have rank at least 2 , so $r>1$. The only possibility for [2, 4] is $r=2$ so $s=16$. 
Since $m / 2=4$, we have $f(c)=b c$ or $f(c)=b c^{-1}$. Suppose that $f(c)=b c^{-1}$. Then $f(c) c=b$ and $f^{4}(x)=f^{3}(c) f^{2}(c) f(c) c x=f^{2}(b) b x=b^{-1} b x=x$, so $f$ has order 4, a contradiction. Suppose instead that $f(c)=b c$, giving $f^{2}(c)=f(b c)=$ $f(b) b c$, and $f^{3}(c)=f^{2}(b) f(b) b c=f(b) c$, and $f^{4}(c)=f(f(b) c)=f^{2}(b) b c=c$, and therefore $f^{4}(x)=f^{3}(c) f^{2}(c) f(c) c x=f(b)^{2} b^{2} c^{4} x$. Since $\left.f\right|_{B}$ has order $2 r=4$, and both $B$ and $C$ have exponent 4, we have $f^{8}(x)=x$, contradicting $s=16$.

We note an important step in the proof is showing that $r$ cannot be even, or in other words, that the group $F$ is 1-good. In fact, Muzychuk [17] has shown this is always the case for abelian 2-groups in case (3).

\section{Questions}

We know that a balanced abelian group has balanced characteristic subgroups and characteristic quotients. What about the unbalanced case?

Question 7.1. If the finite abelian group A has a regular Cayley map, must any characteristic subgroup of A also have a regular Cayley map? Must any quotient of A by a characteristic subgroup?

When we began this work, we had a difficult time finding abelian 2-groups that were 4-good, but not 2-good or 1-good. Muzychuk's characterization explains why: the smallest example has rank 36 . In general, one can ask

Question 7.2. Given a finite 2-group A, what can one say about the spectrum of $k \geq 0$ such that $A$ is $2^{k}$-good?

Our concern has been determining when an abelian group has a regular Cayley map. Being able to classify all regular Cayley maps for a given group is a different matter. One place to begin is cyclic groups, where number theory will be involved:

Question 7.3. Classify all regular Cayley maps of $\mathbb{Z}_{n}$.

Although our interest has been abelian groups, we have one last general question. We have found that many nonabelian groups have Type II balanced maps, but it seems that Type I balanced maps might not be as common.

Question 7.4. Which nonabelian finite groups have Type I balanced maps?

It is an interesting exercise to find Type I balanced automorphisms for the alternating and symmetric groups.

\section{References}

1. N. Biggs, "Cayley maps and symmetrical maps," Proc. Camb. Phil. Soc. 72 (1972), 381-386.

Springer 
2. W. Bosma, J. Cannon, and C. Playoust, "The MAGMA algebra system I: The user language," J. Symbolic Comput. 24 (1997), 235-265.

3. M. Conder, R. Jajcay, and T. Tucker, "Regular $t$-balanced Cayley maps," J. Combin. Theory Ser. B, to appear.

4. M. Conder and P. Dobcsányi, "Determination of all regular maps of small genus," J. Combin. Theory Ser. B 81 (2001), 224-242.

5. M. Conder and I.M. Isaacs, "Derived subgroups of products of an abelian and a cyclic subgroup," J. London Math. Soc. 69 (2004), 333-348.

6. H. Coxeter and W. Moser, Generators and Relations for Discrete Groups 4th edn., Springer-Verlag, Berlin and New York, 1980.

7. A. Gardiner, R. Nedela, J.Širán̆, and M. Škoviera, "Characterization of graphs which underlie regular maps on closed surfaces," J. London Math. Soc. 59(2) (1999), 100-108.

8. D. Gorenstein, Finite Simple Groups: An Introduction to Their Classification. Plenum Publishing Corp., New York, 1982.

9. J.L. Gross and T.W. Tucker, Topological Graph Theory. Wiley, New York, 1987 (Dover paperback, 2001).

10. M. Hall, The Theory of Groups. Chelsea Publishing Co., New York, 1976.

11. N. Ito, "Über das Produkt von zwei abelschen Gruppen," Math Z. 62 (1955), 400-401.

12. R. Jajcay and J. Širáň, "Skew-morphisms of regular Cayley maps," Discrete Math. 244 (2002), 167-179.

13. C. Li, "On edge-transitive Cayley graphs, rotary Cayley maps on 2-manifolds, and s-arc-transitive graphs," preprint.

14. G. Malle, J. Saxl, and T. Weigel, "Generation of classical groups," Geom. Dedicata 49 (1994), 85-116.

15. P. McMullen, B. Monson, and A.I. Weiss, "Regular maps constructed from linear groups," European J. Combin. 14 (1993), 541-552.

16. M. Muzychuk, "On balanced automorphisms of abelian groups," preprint, 2005.

17. M. Muzychuk, "On unbalanced regular cayley Maps for abelian groups," preprint, 2005.

18. B. Richter, J. Širáň, R. Jajcay, T. Tucker, and M. Watkins, "Cayley maps," J. Combin. Theory Ser. B 95 (2005), 489-545.

19. G. Sabidussi, "On a class of fixed-point free graphs," Proc. Amer. Math. Soc. 9 (1958), 800-804.

20. J. Širáń, "Triangle group representations and constructions or regular maps," Proc. London Math. Soc. 82(3) (2001), 513-532.

21. M. Škoviera and J. Širáń, "Regular maps from Cayley graphs, Part I. Balanced Cayley maps," Discrete Math. 109 (1992), 265-276.

22. M. Škoviera and J. Širáň, "Regular maps from Cayley graphs II. Antibalanced Cayley maps," Discrete Math. 124 (1994), 179-191.

23. T.W. Tucker, "Finite groups acting on surfaces and the genus of a group," J. Combin. Theory Ser. B 34 (1983), 323-333.

24. S. Wilson, "Families of regular graphs in regular maps," J. Combin. Theory Ser. B 85 (2002), 264-289. 